\title{
a-Glucosidase and tyrosinase inhibitory effects of an abietane type diterpenoid taxoquinone from Metasequoia glyptostroboides
}

\author{
Vivek K Bajpai ${ }^{1}$, Yong-Ha Park', MinKyun $\mathrm{Na}^{3^{*}}$ and Sun Chul Kang ${ }^{2^{*}}$
}

\begin{abstract}
Background: Nowadays plant derived natural compounds have gained huge amount of research attention especially in food and medicine industries due to their multitude of biological and therapeutic properties as alternative medicines.

Methods: In this study, a diterpenoid compound taxoquinone, isolated from Metasequoia glyptostroboides was evaluated for its a-glucosidase and tyrosinase inhibitory efficacy in terms of its potent anti-diabetic and depigmentation potential, respectively.

Results: As a result, taxoquinone at the concentration range of $100-3,000 \mu \mathrm{g} / \mathrm{mL}$ and $200-1,000 \mu \mathrm{g} / \mathrm{mL}$ showed potent efficacy on inhibiting a-glucosidase and tyrosinase enzymes by $9.24-51.32 \%$ and $11.14-52.32 \%$, respectively.

Conclusions: The findings of this study clearly evident potent therapeutic efficacy of an abietane diterpenoid taxoquinone isolated from M. glyptostroboides with a possibility for using it as a novel candidate in food and medicine industry as a natural alternative medicine to prevent diabetes mellitus type- 2 related disorders and as a depigmentation agent.
\end{abstract}

Keywords: Metasequoia glyptostroboides, Taxoquinone, a-Glucosidase, Tyrosinase, Terpenoids

\section{Background}

Diabetes mellitus is a metabolic disorder caused by a lack of insulin characterized by hyperglycemia [1]. Diabetes mellitus type 2 is also known as non-insulin-dependent diabetes mellitus which is caused by insulin dysfunction, especially after food intake. The effective treatment for type 2 is to inhibit or delay intestinal carbohydrate digestion. Carbohydrates which are the major components of our daily foods, for instance polysaccharides, are transformed into simple sugars, and then absorbed through the intestine. $\alpha$-Glucosidase, an enzyme located in the small intestine epithelium, catalyzes the cleavage of disaccharides and oligosaccharides to glucose. Glucosidase inhibitors reduce the rate of carbohydrate digestion and delay the carbohydrate absorption from the digestive tract.

\footnotetext{
*Correspondence: mkna@cnu.ac.kr; sckang@daegu.ac.kr

${ }^{3}$ College of Pharmacy, Chungnam National University, Daejeon 305-764, Korea

${ }^{2}$ Department of Biotechnology, Daegu University, Gyeongsan, Gyeongbuk

712-714, Korea

Full list of author information is available at the end of the article
}

Therefore, they have a potential to prevent the development of type 2 diabetes mellitus by lowering the aftermeal glucose levels [2].

Human skin contains four major chromophores including haemoglobin, oxyhaemoglobin, carotenoids and melanin(s) where melanin acts as a dominant component of normal skin color and pigmentation [3]. The epidermis cells in the innermost layer of skin produce melanin upon exposure of ultraviolet radiation leading to tyrosinase mediated melanogenesis [3]. Tyrosinase is a multifunctional copper-containing enzyme, widely distributed in plants and animals which catalyzes the initial step in the formation of the pigment melanin from tyrosine [4]. Hence, tyrosinase is known to be a key enzyme for melanin biosynthesis in plants and animals. Although use of tyrosinase inhibitors such as kojic acid and hydroquinone is gaining increasing attention in the cosmetic industry due to their anti-pigmenting effects, they have found to exert severe causes of skin inflammations. Hence, use of natural plant based secondary metabolite to cure skin diseases especially 
for cosmetic purposes could be a safe and alternative therapy in cosmetic industry to provide lead compounds to serve as natural anti-pigmentation compounds [5].

Phytochemicals confer various health benefits, among them, $\alpha$-glucosidase and tyrosinase inhibitory activities have particularly received intensive attention due to the increasing number of patients suffering from diabetes type 2 and skin disorders. Though synthetic $\alpha$-glucosidase and tyrosinase inhibitors have been used effectively, many doubts have been raised on their safety such as increased toxicity and adverse side effects. Hence, attention has been focused on the effective use of plant based compounds which are less toxic and natural in origin.

Although biological and therapeutic potential of M. glyptostroboides derived compounds has been reported previously [6-8], no report is available on $\alpha$-glucosidase and tyrosinase inhibitory effects of taxoquinone, a diterpenoid from M. glyptostroboides. Hence, the aim of this research is to confirm the therapeutic potential of taxoquinone as a potent $\alpha$-glucosidase and tyrosinase inhibitor.

\section{Methods}

\section{Chemicals and instrument}

Kojic acid, acarbose, sodium azide $\left(\mathrm{NaN}_{3}\right)$ bovine serum albumin, P-Nitrophenyl- $\alpha$-D-glucopyranoside, yeast $\alpha$-glucosidase, mushroom tyrosinase, and 3,4-dihydroxyL-phenylalanine (DOPA) were purchased from (SigmaAldrich, St. Louis, MO, USA). All other reagents used were of high analytical grade. Spectrophotometric measurements were done by using a 96-well micro-plate ELISA reader (Infinite M200, Tecan, Switzerland).

\section{Plant material}

The mature cones (16 22 months old) of M. glyptostroboides were collected from Pohang city, Korea, and identified by the morphological features and the database present in the library at the Department of Biotechnology, Daegu University, Korea. A voucher specimen of M. glyptostroboides cone (DUB-0038) was deposited in the herbarium of College of Engineering, Department of Biotechnology, Daegu University, Korea.

\section{Extraction and isolation of taxoquinone}

Dried cones of $M$. glyptostroboides $(2 \mathrm{~kg}$ ) were milled into powder and then extracted with ethyl acetate at room temperature for 12 days. The extract was evaporated under reduced pressure using a rotary evaporator (EYELA N1000, Japan). The dried ethyl acetate extract (7 g) was subjected to column chromatography over silica gel (mesh 230-400 mesh, Merck, Darmstadt, Germany) and was eluted with hexane-ethyl acetate-methanol solvent system to give 20 fractions. Of the fractions obtained, fraction- 12 was further purified by preparative TLC over silica gel GF254 using hexane-ethyl acetate (1:2) as a mobile phase to give one compound (152 mg) which on the basis of spectral data analysis was characterized as a taxoquinone [6].

\section{Assay of a-glucosidase inhibition}

$\alpha$-Glucosidase inhibitory activity of taxoquinone isolated from M. glyptostroboides was evaluated according to the chromogenic method [9]. Briefly $10 \mu \mathrm{L}$ of test samples at various concentrations $(100,500,1,000,2,000$ and $3,000 \mu \mathrm{g} / \mathrm{mL}$ ) and $50 \mu \mathrm{L}$ of yeast $\alpha$-glucosidase, dissolved in $100 \mathrm{mM}$ phosphate buffer (pH 7.0) (containing $2 \mathrm{~g} / \mathrm{L}$ bovine serum albumin and $0.2 \mathrm{~g} / \mathrm{L} \mathrm{NaN}_{3}$ ) were mixed in 96 well micro-plate and absorbance at $405 \mathrm{~nm}$ was measured for titer at zero time with a micro-plate reader (Tecan, Infinite M200, Mannedorf, Switzerland). After 5 min incubation, $50 \mu \mathrm{L}$ of P-Nitrophenyl- $\alpha$-D-glucopyranoside $(5 \mathrm{mM})$ in the same buffer $(\mathrm{pH} 7.0)$ was used as a substrate solution and incubated for an additional $5 \mathrm{~min}$ at room temperature. Eventually the reaction was terminated by adding $80 \mu \mathrm{L}$ of $0.2 \mathrm{M}$ sodium carbonate solution. Absorbance of the reaction mixture was measured with a micro-plate reader at $405 \mathrm{~nm}$. The increase in absorbance from zero time was measured. Inhibitory activity was expressed as 100 minus relative absorbance difference (\%) of test compounds to absorbance change of the control, while the reaction system without sugiol was served as a control test. The system without $\alpha$-glucosidase was used as blank, and acarbose at various concentrations $(100,500,1,000,5,000$ and $10,000 \mu \mathrm{g} / \mathrm{mL})$ was used as a positive control. Each experiment was conducted in triplicate, and the enzyme inhibitory rate was calculated as follows:

$$
\begin{aligned}
\text { Inhibition }(\%)= & (\text { Control absorption }- \text { Sample absorption }) \\
& / \text { Control absorption } \times 100
\end{aligned}
$$

\section{Assay of tyrosinase inhibition}

The tyrosinase activity of taxoquinone was measured by a previously reported method [10]. Briefly, $100 \mu \mathrm{L}$ of different concentrations $(200,400,600,800$ and $1,000 \mu \mathrm{g} / \mathrm{mL}$ ) of taxoquinone were mixed with $600 \mu \mathrm{L}$ of $0.175 \mathrm{M}$ sodium phosphate buffer $(\mathrm{pH}$ 6.8). Further, $200 \mu \mathrm{L}$ of $10 \mathrm{mM}$ L-DOPA solution (L-3,4-dihydroxyphenyl-alanine) was added to each well. After that, $200 \mu \mathrm{L}$ of tyrosinase (110 units $/ \mathrm{mL}$ in $0.175 \mathrm{M}$ sodium phosphate buffer) was added to the reaction mixture and further incubated at $37^{\circ} \mathrm{C}$ for $2 \mathrm{~min}$. Then after incubation, the amount of dopachrome produced in the reaction mixture was measured at $475 \mathrm{~nm}$ in a 96-well micro-titer plate with a micro-plate reader. Kojic acid $(20,40,100,200$ and $500 \mu \mathrm{g} / \mathrm{mL})$ was used as a positive 
control. The experiment was conducted in triplicate at room temperature, and the enzyme inhibitory rate was calculated as follows:

$$
\begin{aligned}
\text { Inhibition }(\%)= & (\text { Control absorption }- \text { Sample absorption }) \\
& / \text { Control absorption } \times 100
\end{aligned}
$$

\section{Statistical analysis}

All the data were expressed as mean \pm standard deviation of three replicates. Tests of significant differences were determined by one way ANOVA followed by Duncan's test using SAS software (SAS 9.2, SAS), and the values were considered to be significant $(\mathrm{p}<0.05)$.

\section{Results and discussion Identification of taxoquinone}

The ethyl acetate cone extract of M. glyptostroboides after column chromatography over silica gel yielded a pure compound which was obtained as orange needles with a specific melting point (mp $\left.212-214^{\circ} \mathrm{C}\right)$. The ${ }^{1} \mathrm{H}$ NMR spectrum $\left(\mathrm{CDCl}_{3}, 250 \mathrm{MHz}\right)$ of the compound showed an oxygenated methine signal at $\delta_{\mathrm{H}} 4.77$ (1H, ddd, $J=2.2,7.4,9.8 \mathrm{~Hz}, \mathrm{H}-7)$, a hydroxyl group at $\delta_{\mathrm{H}} 3.80(1 \mathrm{H}, \mathrm{d}, J=2.2 \mathrm{~Hz}, 7-\mathrm{OH})$, a tertiary methine signal at $\delta_{\mathrm{H}} 3.14(1 \mathrm{H}$, sept, $J=7.1 \mathrm{~Hz}, \mathrm{H}-15)$, three tertiary methyl signals at $\delta_{\mathrm{H}} 1.33(3 \mathrm{H}, \mathrm{s}, \mathrm{H}-20), 0.92$ $(3 \mathrm{H}, \mathrm{s}, \mathrm{H}-18), 0.90$ (3H, s, H-19), and two secondary methyl signals at $\delta_{\mathrm{H}} 1.20(1 \mathrm{H}, \mathrm{d}, J=7.1 \mathrm{~Hz}, \mathrm{H}-17), 1.18$ $(1 \mathrm{H}, \mathrm{d}, J=7.1 \mathrm{~Hz}, \mathrm{H}-16)$. Further analysis of the ${ }^{1} \mathrm{H}-{ }^{1} \mathrm{H}$ COSY data established the connectivity through $\mathrm{H}-7$ $\left(\delta_{\mathrm{H}} 4.77\right), 7-\mathrm{OH}\left(\delta_{\mathrm{H}} 3.80\right)$, and $\mathrm{H}-6 \mathrm{~b}\left[\delta_{\mathrm{H}} 2.18(1 \mathrm{H}, \mathrm{dd}\right.$, $J=7.4,12.5 \mathrm{~Hz})]$, and through $\mathrm{H}-1 \mathrm{~b}\left[\delta_{\mathrm{H}} 2.65(1 \mathrm{H}, \mathrm{br} \mathrm{d}\right.$, $J=13.0 \mathrm{~Hz})], \mathrm{H}-2 \mathrm{~b}\left[\delta_{\mathrm{H}} 1.71(1 \mathrm{H}\right.$, br qt, $\left.J=13.0,3.0)\right]$, and $\mathrm{H}-1 \mathrm{a}, 2 \mathrm{a}, 3 \mathrm{a}, 3 \mathrm{~b}\left(\delta_{\mathrm{H}} 1.65-1.01, \mathrm{~m}\right)$. In addition, two methyl signals at $\delta_{\mathrm{H}} 1.20(\mathrm{H}-17)$ and $1.18(\mathrm{H}-16)$ correlated with a methine signal at $\delta_{\mathrm{H}} 3.14(\mathrm{H}-15)$, which implicated an abietane-type diterpenoid. Twenty carbon signals including two carbonyl groups at $\delta_{C}$ 189.6 (C-14) and 183.7 (C-11) in the ${ }^{13} \mathrm{C}$ NMR spectroscopic data strongly supported that this compound should be an abietane diterpenoid. On the basis of the interpretation of HMQC and HMBC data, this compound was proposed to be taxoquinone. By comparison of the multiplicity of $\mathrm{H}-7$ and the chemical shifts both in the ${ }^{1} \mathrm{H}$ and ${ }^{13} \mathrm{C}$ NMR data, the structure of this compound (Figure 1) was determined to be taxoquinone [6].

\section{Inhibition of a-glucosidase}

Phytochemicals efficiently contribute human being with health beneficial therapeutic agents of natural origin for their use in developing novel diabetes therapies or biotherapeutic agents [11]. Although there is a huge ongoing interest in the application of plant-based compounds for

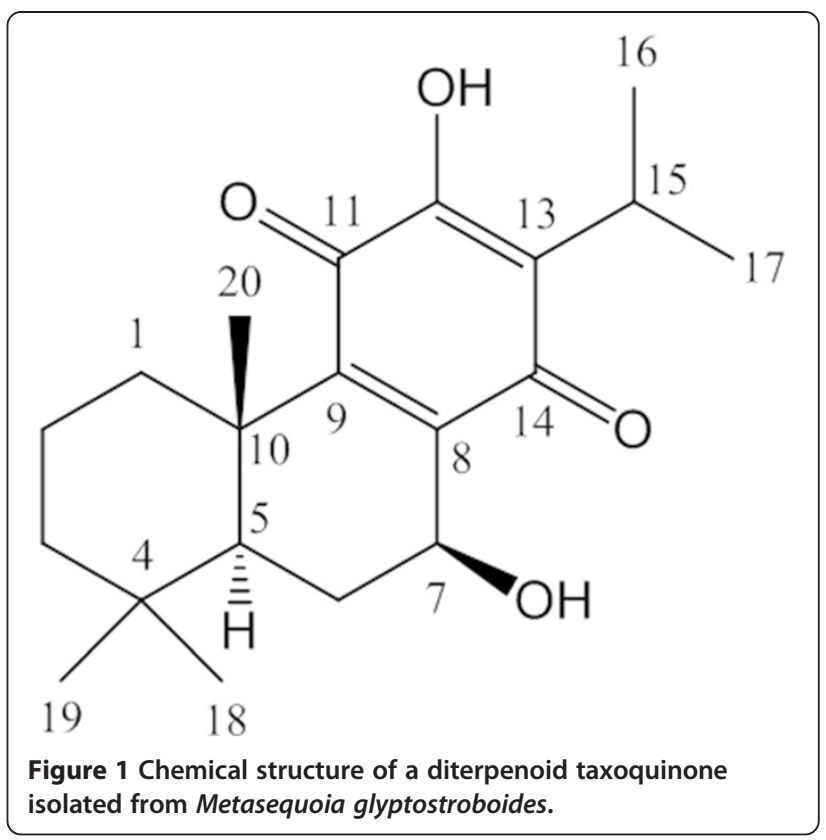

alleviating chronic diseases due to their potent therapeutic and biomedicinal efficacy, the use of phytochemicals within the context of diabetes remains largely unexplored. This has urged the scientists to provide scientific evidences on the development of more effective agents conferring inhibitory effects on intestinal glucosidases. In this regard, inhibition of intestinal $\alpha$-glucosidase to control hyperglycemia is an established strategy $[11,12]$. Hence, to overcome the adversary effects of clinical available synthetic $\alpha$-glucosidase inhibitors, there is still need to develop alternative therapies to inhibit this key enzyme in order to minimize side effects and drug cost efficacy. A number of plant-based natural compounds have been screened for their enzymatic inhibitory activities $[9,11,12]$.

In this assay, the $\alpha$-glucosidase inhibitory activity of taxoquinone was found to be in a concentration dependent manner. The inhibitory effect of taxoquinone on $\alpha$-glucosidase has been demonstrated in Figure 2. The taxoquinone at 100, 500, 1,000, 2,000 and 3,000 $\mu \mathrm{g} / \mathrm{mL}$ showed the inhibition of $\alpha$-glucosidase by 9.24, 14.43, 23.54, 37.43 and $51.32 \%$, respectively. However, standard drug acarbose at 100, 500, 1,000, 5,000 and 10,000 $\mu \mathrm{g} / \mathrm{mL}$ displayed $\alpha$-glucosidase inhibitory effect by $19.16,29.89$, $36.68,57.11$ and $65.52 \%$, respectively. In this assay, both the test compound taxoquinone and acarbose showed inhibitory effect in a dose-dependent manner. Similar findings on $\alpha$-glucosidase inhibitory activity of flavonoid and terpenoid compounds isolated from Agrimonia pilosa were observed by Liu et al. [9]. In addition, sarcoviolins isolated from edible mushroom Sarcodon leucopus were also found to inhibit $\alpha$-glucosidase in vitro [12]. Also the terpenoids including hyptadienic acid isolated from the roots of Potentilla fulgens showed potent inhibitory effect 

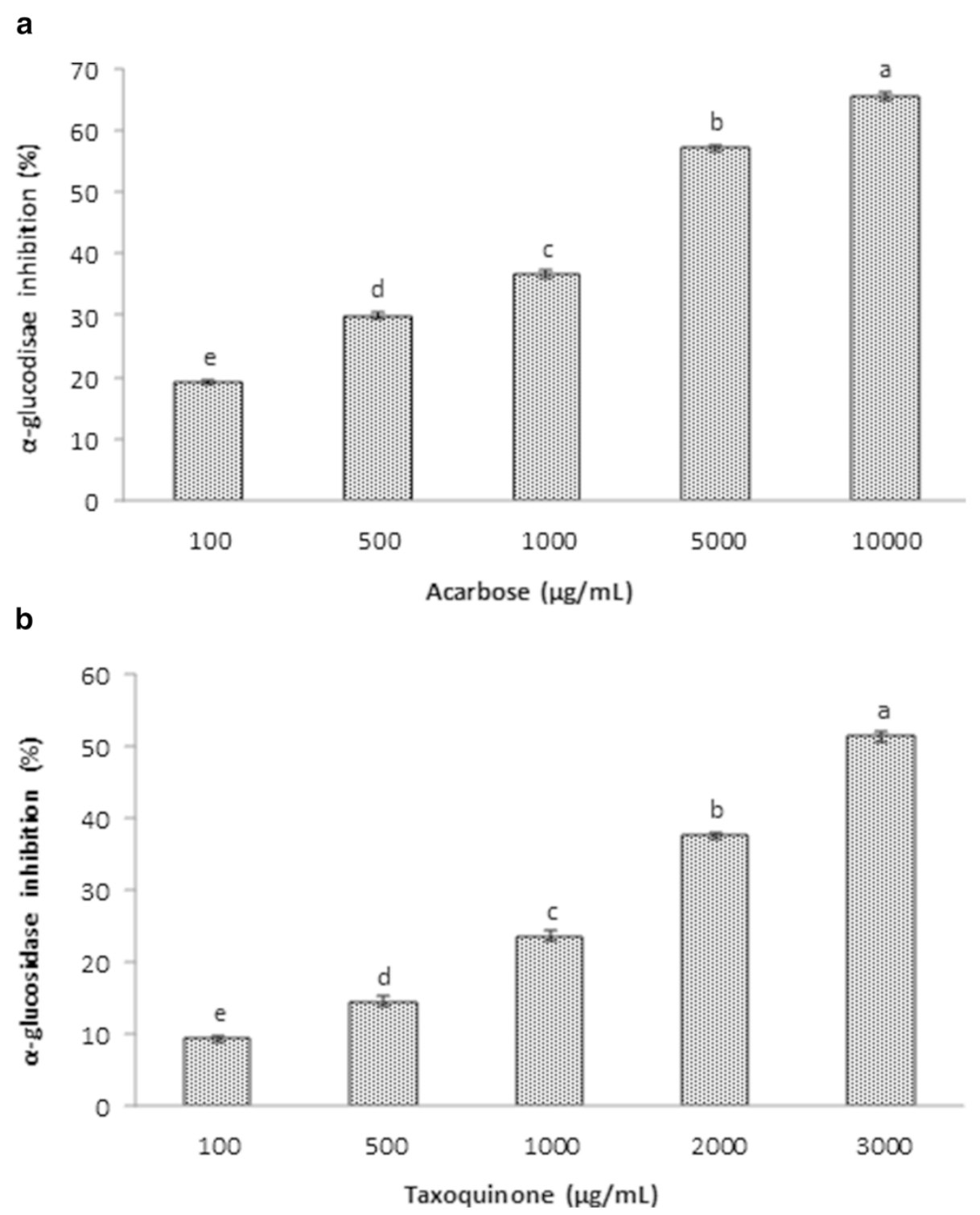

Figure 2 a-Glucosidase inhibitory effect of standard compound acarbose (a) and taxoquinone (b) isolated from Metasequoia glyptostroboides. Data are expressed as mean \pm SD $(n=3)$. Values with different superscripts are significantly different $(p<0.05)$.

on $\alpha$-glucosidase in vitro [13]. Recently, three new phenylpropanoyl amides isolated from the leaves of Piper sarmentosum displayed remarkable $\alpha$-glucosidase inhibitory activities [14].

\section{Inhibition of tyrosinase}

Melanin is a polymerized natural coloring determinant widely distributed in plants, animals and microorganisms. A number of melanins are produced by multi-stepped enzymatic and non-enzymatic oxidation and polymerization processes. The mechanism of tyrosinase inhibition activity may be an important factor in the skin whitening of a cosmetic composition [15]. Melanin biosynthesis steps in the body include L-DOPA by tyrosine as a substrate, followed by its conversion to L-dopaquinone by the successive enzymatic oxidations, leading to take place a reaction of polymerization [15].

The inhibitory activity of taxoquinone on the tyrosinase using a mushroom tyrosinase is demonstrated in Figure 3. In this assay, the taxoquinone at 200, 400, 600, 800 and $1,000 \mu \mathrm{g} / \mathrm{mL}$ showed $11.14,21.33,29.45,38.65$ and $52.32 \%$ of tyrosinase inhibitory effect, respectively. However, the mushroom tyrosinase inhibitory activity of standard compound kojic acid at 20, 40, 100, 200 and $500 \mu \mathrm{g} / \mathrm{mL}$ was found to be 32.41, 43.43, 60.81, 69.05 and $76.54 \%$, respectively. Batubara et al. [16] observed that the phytoconstituent taxifolin and some other flavanonol rhamnosides from $K$. malaccensis also suppressed 
a

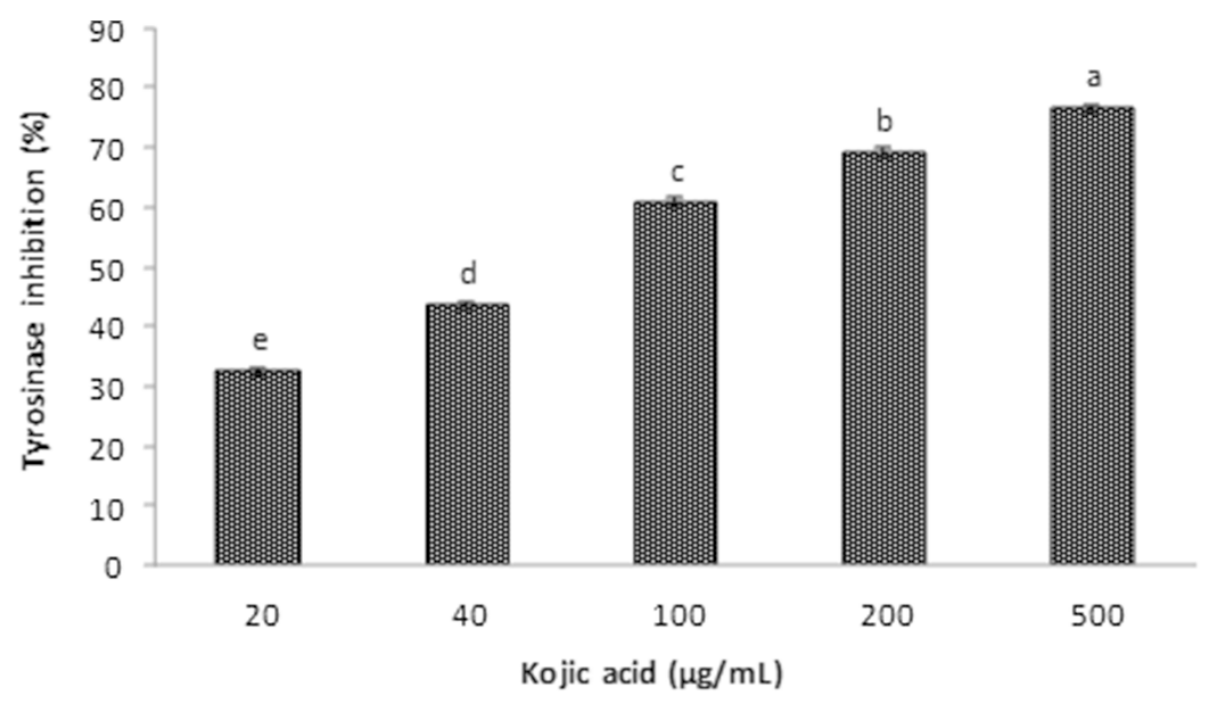

b

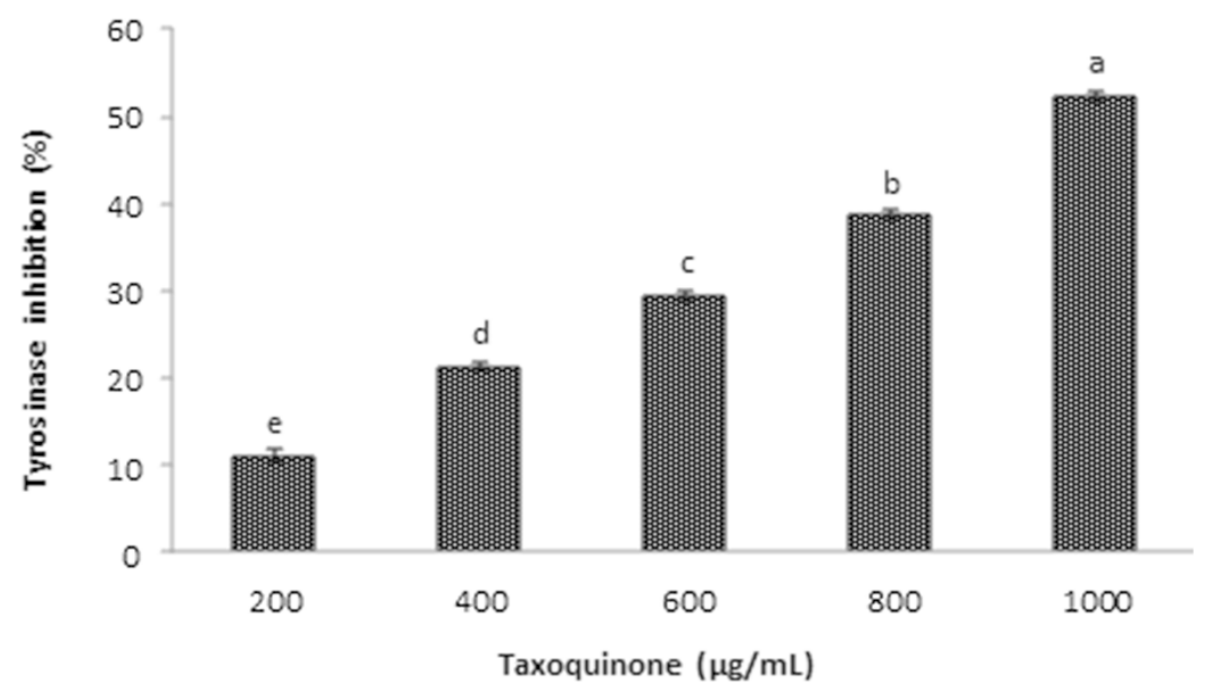

Figure 3 Tyrosinase inhibitory effect of standard compound kojic acid (a) and taxoquinone (b) isolated from Metasequoia glyptostroboides. Data are expressed as mean \pm SD $(n=3)$. Values with different superscripts are significantly different $(p<0.05)$.

tyrosinase activity in the range of 5.86-25.95\%. Piao et al. [17] demonstrated tyrosinase inhibitory effect of some chromones derived from a tropical plant aloe in a dosedependent manner. In addition, the terpenoids isolated from the leaves of Chloranthus tianmushanensis were also found to exhibit tyrosinase inhibitor effect in a dose-dependent manner [18]. Similar findings on dosedependent tyrosinase inhibitory activity of taxoquinone were observed in this study. Since it has been shown that tyrosinase inhibitors can repress the conversion of tyrosine to DOPA, dopaquinone and subsequently melanin, various tyrosinase inhibitors have been isolated and studied as potential candidates to decrease melanin content [19].

\section{Conclusions}

In this research, a diterpenoid compound taxoquinone isolated from M. glyptostroboides demonstrated a considerable amount of $\alpha$-glucosidase and tyrosinase inhibitory effects in vitro which may have potential to reduce after meal blood glucose levels and to maintain healthy skin by serving as a skin depigmentation agent. Based on the finding of this study, it can be concluded that taxoquinone in terms of its potent hypoglycemic and skin-whiting efficacy makes it to be a molecule of choice for using in healthcare and drug therapies in the treatment of infectious diseases. However, further studies are needed to demonstrate a precise mode of action of taxoquinone to confirm its in vivo anti-diabetic and depigmentation potential. 


\section{Competing interests}

The authors declare that they have no competing interests.

\section{Authors' contributions}

VKB: Collected plant sample material, prepared extract, isolated terpenoid compound, carried out assays and prepared a draft of manuscript. MN: Done spectral data analysis and structure determination. YHP: Conceived and designed the study. SCK and VKB: Conceived and revised the manuscript. Finally both VKB and SCK read and approved the final manuscript. All authors read and approved the final manuscript.

\section{Author details}

${ }^{1}$ Department of Applied Microbiology and Biotechnology, School of Biotechnology, Yeungnam University, Gyeongsan, Gyeongbuk 712-749, Korea. 'Department of Biotechnology, Daegu University, Gyeongsan, Gyeongbuk 712-714, Korea. ${ }^{3}$ College of Pharmacy, Chungnam National University, Daejeon 305-764, Korea.

Received: 1 October 2014 Accepted: 18 March 2015

Published online: 26 March 2015

\section{References}

1. Fatmawatia S, Shimizua K, Kondoa R, Ganoderol B. A potent a-glucosidase inhibitor isolated from the fruiting body of Ganoderma lucidum. Phytomedicine. 2011;18:1053-5.

2. Liu L, Deseo MA, Morris C, Winter KM, Leach DN. Investigation of a-glucosidase inhibitory activity of wheat bran and germ. Food Chem. 2011;126:553-61.

3. Momtaz S, Mapunya BM, Houghton PJ, Edgerly C, Hussein A, Naidoo S, et al. Tyrosinase inhibition by extracts and constituents of Sideroxylon inerme $\mathrm{L}$. stem bark, used in South Africa for skin lightening. J Ethnopharmacol. 2008;119:507-12.

4. Kim YJ, Uyama H. Tyrosinase inhibitors from natural and synthetic sources: structure, inhibition mechanism and perspective for the future. CMLS. 2005:62:1707-23.

5. Pieroni A, Cassandra L, Villanelli M, Mangino P, Sabbatini G, Boccetti T, et al Ethnopharmacognostic survey on the natural ingredients in folk cosmetics, cosmeceuticals and remedies skin disease in the inland Marches, Central-Eastern Italy. J Ethnopharmacol. 2004;91:331-44.

6. Bajpai VK, Na MK, Kang SC. The role of bioactive substances in controlling foodborne pathogens derived from Metasequoia glyptostroboides Miki ex Hu. Food Chem Toxicol. 2010:48:1945-9.

7. Bajpai VK, Kang SC. Isolation and characterization of biologically active secondary metabolites from Metasequoia glyptostroboides Miki Ex Hu. J Food Safety. 2011;31:276-83.

8. Bajpai VK, Kang SC. Antimycotic potential of a diterpenoid taxoquinone against Candida species isolated from Metasequoia glyptostroboides. Bangladesh J Pharmacol. 2014;9:154-60.

9. Liu X, Zhu L, Tan J, Zhou X, Xiao L, Yang X, et al. Glucosidase inhibitory activity and antioxidant activity of flavonoid compound and triterpenoids compound from Agrimonia Pilosa Ledeb. BMC Comp Alt Med. 2014;14:12-6.

10. Fawole OA, Makunga NP, Opara UL. Antibacterial, antioxidant and tyrosinase-inhibition activities of pomegranate fruit peel methanolic extract. BMC Comp Alt Med. 2012;12:202-11.

11. Tiwari AK, Viswanadh V, Gowri PM, Ali AZ, Radhakrishnan SVS, Agawane SB, et al. Oleanolic acid - an a-Glucosidase inhibitory and antihyperglycemic active compound from the fruits of Sonneratia caseolaris. J Med Arom Plants. 2010;1:19-23.

12. Ma K, Han J, Bao L, Wei T, Liu H. Two sarcoviolins with antioxidative and a-glucosidase inhibitory activity from the edible mushroom Sarcodon leucopus collected in Tibet. Nat Prod. 2014;77:942-7.

13. Kumar D, Ghosh R, Pal BC. a-Glucosidase inhibitory terpenoids from Potentilla fulgens and their quantitative estimation by validated HPLC method. J Fun Food. 2013;5:1135-41.

14. Damsud T, Adisakwattana S, Phuwapraisirisan P. Three new phenylpropanoyl amides from the leaves of Piper sarmentosum and their a-glucosidase inhibitory activities. Phtochem Lett. 2013;6:350-4.

15. An BJ, Kwak JH, Park JM, Lee JY, Park TS, Lee JT, et al. Inhibition of enzyme activities and the antiwrinkle effect of polyphenol isolated from the persimmon leaf (Diospyros kaki folium) on human skin. Dermatol Surg. 2005;31:848-54.
16. Batubara I, Kuspradini H, Mitsunaga T. Anti-acne and tyrosinase inhibition properties of taxifolin and some flavanonol rhamnosides from Kempas (Koompassia malaccensis). Wood Res J. 2010;1:45-9.

17. Piao LZ, Park HR, Park YK, Lee SK, Park JH, Park MK. Mushroom tyrosinase inhibition activity of some chromones. Chem Pharm Bull. 2002;50:309-11.

18. Wu B, Chen J, Qu H, Cheng Y. Complex Sesquiterpenoids with tyrosinase inhibitory activity from the leaves of Chloranthus tianmushanensis. J Nat Prod. 2008;71:877-80.

19. Khan MTH, Khan SB, Ather AL. Tyrosinase inhibitory cycloartane type triterpenoids from the methanol extract of the whole plant of Amberboa ramosa Jafri and their structure-activity relationship. Bioorg Med Chem. 2006;14:938-43.

\section{Submit your next manuscript to BioMed Central and take full advantage of:}

- Convenient online submission

- Thorough peer review

- No space constraints or color figure charges

- Immediate publication on acceptance

- Inclusion in PubMed, CAS, Scopus and Google Scholar

- Research which is freely available for redistribution 